\title{
Anaesthetic management of a patient with Atrial Septal Defect with Pulmonary Hypertension posted for Total Abdominal Hysterectomy: a Case Report
}

\author{
Danish Omair ${ }^{1}$, S. Padmanabha ${ }^{2}$ \\ ${ }^{1,2,}$ Yenepoya Medical College, Yenepoya University, Deralakatte, Mangalore-575018, India.
}

\begin{abstract}
An asymptomatic 52 year old lady with Atrial Septal Defect of Ostium Secundum type with mild Pulmonary Hypertension was admitted in our hospital with complains of menorrhagia. After a thorough preoperative evaluation, adequate preparation she underwent total abdominal hysterectomy under combined epidural and general anaesthesia. By providing proper intraoperative and postoperative care, patient was managed without any complication and was discharged home within a week.
\end{abstract}

Keywords: Atrial Septal Defect, Pulmonary Hypertension, Total Abdominal Hysterectomy, General Anaesthesia, Epidural Anaesthesia.

\section{Introduction}

Atrial Septal Defect (ASD) is a common cardiac anomaly in children occurring most commonly in females. The most common atrial septal defect involves fossa ovalis, is midseptal in location and is of the ostium secundum type ${ }^{1}$. Based on the portion of atrial septum that has failed to develop normally, ASDs are anatomically classified into four types: ostium secundum ( $85 \%)$, ostium primum (10\%), sinus venosus $(5 \%)$, and coronary sinus defects(rare). Spontaneous closure occurs by 18 months in almost all patients born with ASDs $<3 \mathrm{~mm}$ diameter and $80 \%$ of those with defects $3-8 \mathrm{~mm}$. Defects with diameters $>8 \mathrm{~mm}$ rarely close spontaneously and may require surgery later in life ${ }^{2}$. The majority of ASDs are detected in childhood although a significant minority is only diagnosed in adult life. Initially right ventricular compliance is substantially greater than the left ventricle and this is associated with the development of a left-to-right shunt. Pulmonary blood flow is increased and pulmonary hypertension develops with increasing age. A consequence of these physiological changes is dilatation of both left and right atria, the right ventricle and the pulmonary arteries in order to accommodate the increased blood volume. Ultimately either the right ventricle fails, or right ventricular compliance falls, resulting in a reduction in the magnitude of shunting, or even flow reversal ${ }^{3}$. Presentation depends upon the magnitude of the left to right shunt. Effort dyspnea is seen in $30 \%$ of patients by the third decade and more than $75 \%$ of patients by the fifth decade. Atrial arrhythmias (atrial fibrillation and flutter) are common and are secondary to atrial dilatation. Clinical examination reveal fixed splitting of the second heart sound coupled with a pulmonary flow murmur. The classically described electrocardiogram of an ASD is right bundle branch block (RBBB) and axis deviation (right for secundum and left for primum) ${ }^{4}$. Complications of ASD in adults include: paradoxic emboli, effort dyspnea, atrial tachyarrhythmia, right sided heart failure with pregnancy, pulmonary hypertension and mitral insufficiency ${ }^{5}$.

\section{Case History}

A 52 year old female G2P2 who is a known hypertensive was admitted in our hospital with a history of menorrhagia. She also has a history of exertional dyspnea NYHA grade II and repeated respiratory tract infections. She is on Tab Atenolol, Tab Torsemide and Spironolactone daily.no history of any past surgery or anaesthesia.

On examination her pulse rate was 80 beats/minute, regular and good volume, non-invasive blood pressure was $130 / 70 \mathrm{mmHg}$. On auscultation, normal vesicular breath sounds were heard, pulmonary area revealed ejection systolic murmur.12 lead electrocardiogram showed Right Bundle Branch Block, Right Axis Deviation and rSR' pattern in lead V1. Echocardiogram showed Ostium Secundum type ASD (15mm) with left to right shunt, mild right atrium and right ventricular dilatation and mild Pulmonary Hypertension. Chest X-ray revealed increased broncho-vesicular markings and cardiomegaly. All the other blood investigation were within normal limits. Ultrasound of the abdomen revealed fibroid uterus and she was planned for Total Abdominal Hysterectomy.

\section{Anaesthetic Management}

After a detailed pre-anaesthetic evaluation and investigations, written informed consent was obtained from the patient. Patient was kept nil per orally for $6 \mathrm{hrs}$. Oral hypertensives and diuretics were continued on the 
day of the surgery. Tab Ranitidine $150 \mathrm{mg}$, Inj Glcopyrrolate $0.2 \mathrm{mg}$ IM and Tab Lorazepam $1 \mathrm{mg}$ was given orally as premedication 1 hour before surgery. Antibiotic prophylaxis Inj Ampicillin $2 \mathrm{Gm}$ IV was given preoperatively. Venous access secured by an $18 \mathrm{G}$ intravenous cannula on the dorsum of the left hand and patient preloaded with $500 \mathrm{ml}$ Ringer Lactate solution. Routine monitors were connected like pulse oximetry, noninvasive blood pressure and ECG. Basal values of vitals were recorded. For epidural anaesthesia, parts were painted and drapped under strict aseptic precautions, patient lying on left lateral decubitus position. 18G Touhys needle was used in L1-2 interspace with loss of resistance technique, loss of resistance felt at $4 \mathrm{cms}$ and catheter fixed at $9 \mathrm{cms}$. Test dose of $2 \%$ lignocaine with adrenaline $3 \mathrm{ml}$ was given after aspiration.

General anaesthesia was administered, with all precautions to avoid systemic hypotension and air entrainment through the venous circulation. Patient was pre-oxygenated with $100 \%$ oxygen for 3 mins using Bain's circuit. Induced with Inj. Fentanyl $50 \mathrm{ug}$, Inj. Propofol $120 \mathrm{mg}$ and Inj. Vecuronium $5 \mathrm{mg}$. Used $7.5 \mathrm{ID}$ endotracheal tube was secured, bilateral air entry was confirmed and end- tidal carbon dioxide monitor was connected. Controlled ventilation was maintained using air, oxygen and Sevoflurane.. Nitrous oxide was avoided because of a risk of paradoxical air embolism. For analgesia intra-operatively $0.25 \%$ Inj Bupivacaine and Inj fentanyl $1 \mathrm{ug} / \mathrm{ml}$ was used. Intra-operatively one episode of bradycardia was seen during the surgery which was treated with Inj Atropine $0.6 \mathrm{mg} \mathrm{IV}$, rest all parameters were normal. Surgery lasted for $2 \mathrm{hrs} 30$ mins. Intraoperatively intake was $1000 \mathrm{ml} \mathrm{RL}$ and $200 \mathrm{ml}$ DNS. Urine output was $300 \mathrm{~mL}$ intra-operatively and blood loss approximately $150 \mathrm{~mL}$. Patient was reversed with Inj Neostigmine $2.5 \mathrm{mg}$ and Inj Glycopyrollate 0.5 mg IV. Post-extubation patient was comfortable, breathing spontaneously and obeying commands. Postoperative vitals were stable. For post-operative analgesia $0.125 \%$ Bupivacaine with 100 ug Fentanyl infusion in $50 \mathrm{ml}$ syringe was loaded, epidural infusion was started at $4 \mathrm{ml} / \mathrm{hr}$. Epidural analgesia was provided for 48 hours post-operatively and patient was comfortable. Post-operatively maintenance fluid was administered as per the urine output.

\section{Discussion}

ASD occurs in 1 in 1500 live births and comprises $6 \%-10 \%$ of all congenital heart diseases. An ASD is an opening in the interatrial septum. ASD classification is based on ASD location relative to the fossa ovalis and developmental anatomy. It is also classified according to the defect size. ASD is one of the most common congenital heart diseases and a progressive disorder resulting in reversal of the shunt.

Anatomic classification includes:

1. Patent Foramen Ovale (PFO): it is a small interatrial communication in the foramen ovale region, with no deficiency of the septum primum or septum secundum. Foramen ovale is a normal communication in fetal life that closes at birth when left atrial pressure exceeds right atrial pressure.

2. Ostium Primum ASD: it is a crescent shaped defect in the inferior portion of atrial septum above the atrioventricular valve.

3. Ostium Secundum ASD: it is confined to the fossa ovalis region and is a deficiency of the spetum primum. It is the most common type of ASD. Occasionally, these defects are part of the spectrum of Holt-Oram syndrome, which also includes limb anomalies.

4. Sinus Venosus ASD: this defect occurs high in the septum in the sinus venosus region, near the orifice of the superior vena cava. It may occur in the inferior sinoatrial junction region, near the inferior vena cava orifice.

5. Coronary Sinus ASD: is a defect in the atrial wall separating the left atrium from the coronary sinus. It allows blood to shunt from the left atrium to the right atrium via the coronary sinus.

6. Common Atrium: also known as single atrium, represents complete absence of an interatrial septum.

Classification based on defect size:

Small defects $(<3 \mathrm{~mm})$ are expected to close spontaneously. $80 \%$ of medium-sized defects $(3-8 \mathrm{~mm})$ close spontaneously. Defects larger than $8 \mathrm{~mm}$ most probably will not close ${ }^{6}$.

Adverse effects of ASD due to persistently increased pulmonary blood flow may result in following pathophysiological changes:

1) Increase in pulmonary vascular resistance (PVR) and development of pulmonary arterial hypertension $(\mathrm{PAH})$

2) Cardiomegaly and cardiac remodeling

3) Arrhythmias: atrial, ventricular and other types of dysrhythmias can occur in patients with left-toright cardiac shunt. These rhythm disturbances are either due to intrinsic nature of the anomaly or 
to morphological changes secondary to blood overloading the right side of heart, or surgical palliation.

4) Myocardial ischemia . ${ }^{2}$

\section{Conclusion}

Septum secundum type of ASD do not close spontaneously and are usually progressive in nature, resulting in Eisenmenger's syndrome, however, the patient mentioned above did not show any evidence of complications of ASD. With the thorough pre-operative assessment, proper preparation and providing good intra operative and post-operative analgesia, non-cardiac surgeries like hysterectomy was performed safely. The mortality and morbidity will increase once the patient develops severe pulmonary hypertension.

[1] Harrison principles of internal medicine, $17^{\text {th }}$ edition: 1459

[2] Alan D Kaye, Tyler B Stout, Ira W Padnos, Brian G Schwartz, Amir R Baluch, Charles J Fox et al. Left-to-right cardiac shunt: Perioperative anesthetic considerations. MEJ Anesth. 21(6) 2012

[3] AT Lovell. Anesthetic implications of grown up congenital heart disorders. Br J Anaesth. 2004;93:129-139

[4] Patrick A Calvart, Andrew A klein. Anesthesia for percutaneous closure of ASD. Continuing Education in Anaesthesia, Critical Care and Pain. 2008,Vol 8,Nol

[5] Kaplan: Essentials of cardiac anaesthesia, $1^{\text {st }}$ edition

[6] Pediatric cardiac anaesthesia $4^{\text {th }}$ edition 2005 